\title{
Investigating the Evolution of the Dual AGN System ESO 509-IG066
}

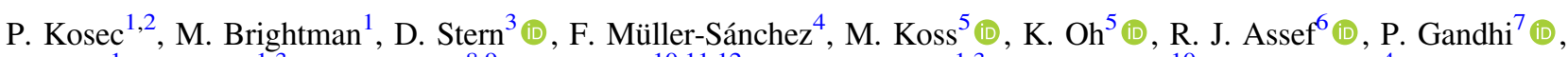

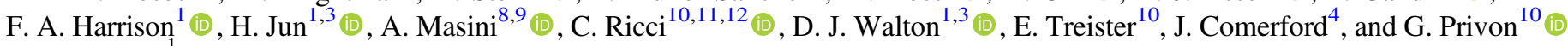 \\ ${ }^{1}$ Cahill Center for Astrophysics, California Institute of Technology, 1216 East California Boulevard, Pasadena, CA 91125, USA \\ ${ }^{2}$ Institute of Astronomy, Madingley Road, CB3 OHA Cambridge, UK \\ ${ }^{3}$ Jet Propulsion Laboratory, California Institute of Technology, Pasadena, CA 91109, USA \\ ${ }^{4}$ Department of Astrophysical and Planetary Sciences, University of Colorado, Boulder, CO 80309, USA \\ ${ }^{5}$ Institute for Astronomy, Department of Physics, ETH Zurich, Wolfgang-Pauli-Strasse 27, CH-8093 Zurich, Switzerland \\ ${ }^{6}$ Núcleo de Astronomía de la Facultad de Ingeniería, Universidad Diego Portales, Av. Ejército Libertador 441, Santiago, Chile \\ ${ }^{7}$ Department of Physics and Astronomy, University of Southampton, Highfield, Southampton SO17 1BJ, UK \\ 8 INAF Osservatorio Astronomico di Bologna, via Ranzani 1, I-40127 Bologna, Italy \\ ${ }_{10}^{9}$ Dipartimento di Fisica e Astronomia (DIFA), Universitá di Bologna, viale Berti Pichat 6/2, I-40127 Bologna, Italy \\ ${ }^{10}$ Instituto de Astrofísica, Facultad de Física, Pontificia Universidad Católica de Chile, Casilla 306, Santiago 22, Chile \\ ${ }^{11}$ Kavli Institute for Astronomy and Astrophysics, Peking University, Beijing 100871, China \\ ${ }^{12}$ Chinese Academy of Sciences South America Center for Astronomy and China-Chile Joint Center for Astronomy, \\ Camino El Observatorio 1515, Las Condes, Santiago, Chile \\ Received 2017 January 9; revised 2017 September 13; accepted 2017 October 5; published 2017 November 28
}

\begin{abstract}
We analyze the evolution of the dual active galactic nucleus (AGN) in ESO 509-IG066, a galaxy pair located at $z=0.034$ whose nuclei are separated by $11 \mathrm{kpc}$. Previous observations with XMM-Newton on this dual AGN found evidence for two moderately obscured $\left(N_{\mathrm{H}} \sim 10^{22} \mathrm{~cm}^{-2}\right)$ X-ray luminous $\left(L_{\mathrm{X}} \sim 10^{43} \mathrm{erg} \mathrm{s}{ }^{-1}\right)$ nuclear sources. We present an analysis of subsequent Chandra, NuSTAR, and Swift/XRT observations that show one source has dropped in flux by a factor of 10 between 2004 and 2011, which could be explained by either an increase in the absorbing column or an intrinsic fading of the central engine, possibly due to a decrease in mass accretion. Both of these scenarios are predicted by galaxy merger simulations. The source that has dropped in flux is not detected by NUSTAR, which argues against absorption, unless it is extreme. However, new Keck/LRIS optical spectroscopy reveals a previously unreported broad $\mathrm{H} \alpha$ line that is highly unlikely to be visible under the extreme absorption scenario. We therefore conclude that the black hole in this nucleus has undergone a dramatic drop in its accretion rate. From AO-assisted near-infrared integral-field spectroscopy of the other nucleus, we find evidence that the galaxy merger is having a direct effect on the kinematics of the gas close to the nucleus of the galaxy, providing a direct observational link between the galaxy merger and the mass accretion rate onto the black hole.
\end{abstract}

Key words: galaxies: active - galaxies: individual (ESO 509-IG066) - galaxies: nuclei - galaxies: Seyfert - X-rays: galaxies

\section{Introduction}

Interactions between galaxies are predicted to cause increased nuclear activity (e.g., Sanders et al. 1988; Hernquist 1989). Massive gas flows triggered by gravitational interaction and resulting tidal forces can potentially fuel central supermassive black holes, creating luminous active galactic nuclei (AGNs). This has been shown observationally in large statistical samples of galaxy pairs, where the AGN fraction and AGN luminosity have both been shown to increase as the separation between the galaxies decreases, peaking at $\sim 10 \mathrm{kpc}$ (Alonso et al. 2007; Woods \& Geller 2007; Ellison et al. 2011; Silverman et al. 2011; Koss et al. 2012; Satyapal et al. 2014). In addition, it is naturally expected that such a gas buildup in the nucleus will not only fuel the growth of the supermassive black hole, but obscure it as well, at Compton-thick levels $\left(N_{\mathrm{H}}>10^{24} \mathrm{~cm}^{-2}\right.$, Hopkins et al. 2005). Indeed, this has been shown recently with a sample of interacting galaxies at $z \sim 1$ where galaxies that exhibit evidence for a merger or interaction were more likely to host a Compton-thick AGN than a less obscured one (Kocevski et al. 2015; Ricci et al. 2017).

However, while both observations and simulations of galaxy mergers find that AGN obscuration increases in galaxy mergers, simulations have also shown that large fluctuations in the mass accretion rate onto the black hole are also to be expected (e.g., Di Matteo et al. 2005; Hopkins et al. 2006), especially during the later stages of the merger (e.g., Gabor et al. 2016). Observationally, however, it is challenging to distinguish between changes in accretion rate and changes in absorption, since both lead to changes in the observed flux (e.g., Rivers et al. 2015a; Gandhi et al. 2017).

In this paper we study a local pair of interacting galaxies, ESO 509-IG066 (Figure 1), located at $z=0.034\left(D_{\mathrm{L}} \sim\right.$ $150 \mathrm{Mpc})$. The galaxy pair was chosen for this study as part of a NUSTAR program to observe Swift/BAT-detected AGNs (Harrison et al. 2013). The nuclei of the two galaxies are separated by $16^{\prime \prime}$ on the sky, which at this redshift implies a physical projected separation of $10.9 \mathrm{kpc}$ (assuming $H_{0}=$ $67.8 \mathrm{~km} \mathrm{~s}^{-1} \mathrm{Mpc}^{-1}, \Omega_{\mathrm{m}}=0.308$, and $\Omega_{\Lambda}=0.692$, Planck Collaboration et al. 2016). The galaxies are aligned in the east-west direction where the R.A. and decl. of the nuclei are $203^{\circ} .6653,-23^{\circ} .4468$, henceforth known as the "Western source" and $203^{\circ} .6700,-23^{\circ} .4461$, henceforth known as the "Eastern source." Guainazzi et al. (2005, hereafter G05) analyzed the galaxy pair using XMM-Newton data from 2004 and reported that both galaxies host luminous nuclear X-ray sources with luminosities of $\sim 10^{43} \mathrm{erg} \mathrm{s}^{-1}$. They found that the Western source is a moderately obscured AGN with a 


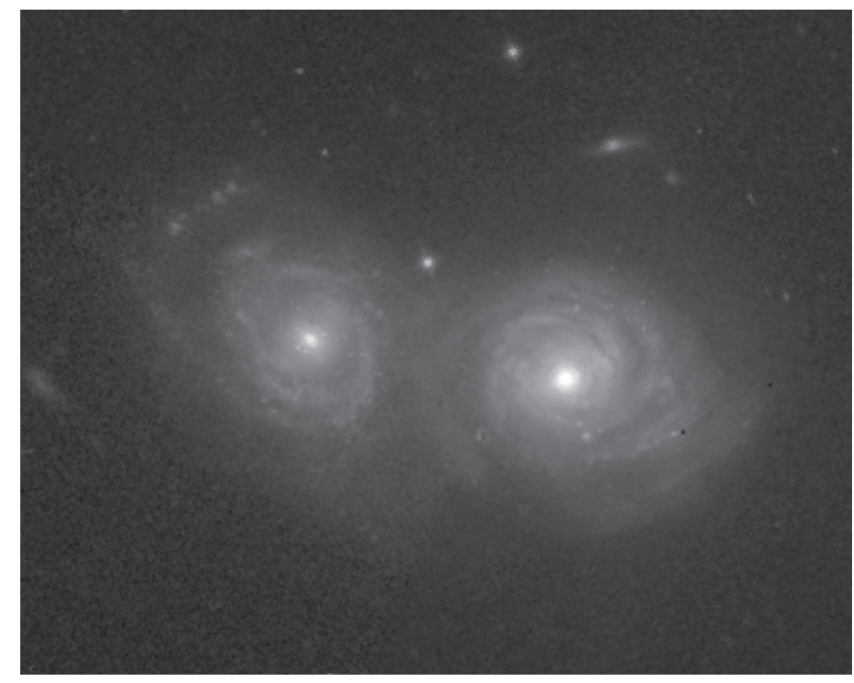

Figure 1. HST/WFPC2 F606W image of the galaxy pair ESO 509-IG066 from Malkan et al. (1998). The Eastern galaxy is on the left and the Western galaxy is on the right. The image is $50^{\prime \prime} \times 40^{\prime \prime}$.

column density of $\sim 10^{23} \mathrm{~cm}^{-2}$, while the Eastern source is almost unobscured with the column density less than $10^{22} \mathrm{~cm}^{-2}$. While the Western source is very weak in the very soft X-ray band $(0.5-2 \mathrm{keV})$, it outshines the Eastern source in harder bands $(2.0-10.0 \mathrm{keV})$. The system has also been detected by Swift/BAT (Cusumano et al. 2010; Baumgartner et al. 2013) with a 14-195 keV flux of $1.4 \times$ $10^{-11} \mathrm{erg} \mathrm{cm}^{-2} \mathrm{~s}^{-1}$, although with a PSF of $10^{\prime}$, Swift/BAT cannot resolve these two nuclei. Furthermore, ESO 509IG066 was detected by MAXI/GSC (Hiroi et al. 2011) with a 4-10 keV flux of $1.7 \times 10^{-11} \mathrm{erg} \mathrm{cm}^{-2} \mathrm{~s}^{-1}$.

In this paper we reanalyze the XMM-Newton data from 2004 and add new results from the Chandra, NuSTAR, and Swift observations. In addition to the X-ray data, we use data from the Catalina Sky Survey (Drake et al. 2009) and the Wide-field Infrared Survey Explorer (WISE, Wright et al. 2010) to compare the X-ray variations with the variability in the optical and infrared (IR). Furthermore, we present new Keck/LRIS optical spectroscopic observations of the galaxies and a Keck/ OSIRIS near-IR integral-field spectroscopic observation of the Western nucleus that yield insights into the system.

The structure of this paper is as follows. In Section 2 we describe the observational data used and the data reduction. Section 3 briefly summarizes our X-ray spectral fitting methods and results, followed by results from optical and IR analysis listed in Section 4. We present new Keck/LRIS optical spectroscopy and Keck/OSIRIS near-IR integral-field spectroscopy in Section 5. We discuss our results in Section 6 and conclude in Section 7.

\section{Observations}

ESO 509-IG066 has been observed by XMM-Newton, Chandra, NuSTAR, and Swift, and the NuSTAR and Swift observations were simultaneous. Figure 2 presents the X-ray images of the system from each of the observatories and Table 1 summarizes the basic observational data. The following sections discuss the processing of each of these X-ray data sets, as well as ancillary data sets at optical and IR wavelengths.

\subsection{XMM-Newton}

XMM-Newton (Jansen et al. 2001) EPIC-pn (Strüder et al. 2001) data were reduced using SAS v14.0, selecting events from a circular region of radius $60^{\prime \prime}$ centered on the galaxy pair corresponding to a $\sim 90 \%$ encircled energy fraction. EPIC-MOS data were not considered due to their lower hard $\mathrm{X}$-ray sensitivity. A period of high background at the beginning of the observation was filtered out, leaving $7.9 \mathrm{ks}$ of science data. Background spectra were extracted from a nearby circular region of $75^{\prime \prime}$ radius on the same chip as the galaxies. Initially, both of the nuclei were extracted in a single spectrum. During a subsequent analysis, we also extracted a spectrum for each of the two objects. For this individual analysis we used circular regions of radius $8^{\prime \prime}$ for the Eastern source and $7^{\prime \prime}$ for the Western source, respectively. Spectra were grouped with a minimum of 20 counts per bin. We carried out spectral fitting in the $0.2-10.0 \mathrm{keV}$ energy range.

\subsection{Chandra}

The Chandra (Weisskopf 2000) data of the Eastern and Western sources were extracted using the CIAO (v4.7, CALDB v4.6.5) tool SPECEXTRACT, from circular regions with a radius of $5^{\prime \prime}$. A larger circular region on the same chip as the galaxies was used to extract the background spectrum. The spectrum of the Western source (the brighter one) was grouped to at least 10 counts per bin and the spectrum of the Eastern source was binned to at least 5 counts per bin. Counts at energies below 0.5 and above $7.5 \mathrm{keV}$ were ignored, as the efficiency of the instrument drops quickly when outside of this energy range.

\subsection{NUSTAR}

The NUSTAR (Harrison et al. 2013) raw data were reduced using the NUSTARDAS v 1.5.1 software. Initially the events were cleaned and filtered with the nupipeline script with standard parameters, then the nuproducts procedure was used to extract spectra and the corresponding response and auxiliary files. A single spectrum was extracted for the galaxy pair because the size of the PSF of NuSTAR $\left(\sim 60^{\prime \prime}\right.$, Madsen et al. 2015) is larger than the separation of the galaxies. The spectra were extracted from circular regions centered on the peak of emission and with specific radii to maximize the signalto-noise ratio. The background spectra were obtained from regions chosen to cover as much area as possible on the same detector as the source while avoiding the source itself and its point-spread function. Data from both focal plane modules (FPMA and FPMB) were extracted and used in simultaneous fitting without coadding. Both $N u S T A R$ spectra were grouped by at least 20 counts per bin using the HEASARC tool GRPPHA. We ignore channels below $3 \mathrm{keV}$, as the calibration at lower energies is uncertain, and we ignore channels above the $79 \mathrm{keV}$ cutoff that results from absorption in the mirror coating.

\subsection{Swift}

The Swift/XRT (Gehrels et al. 2004; Burrows et al. 2005) observation was taken simultaneously with the NuSTAR observation. The data were preprocessed and the spectrum was extracted using automatic routines XRTPIPELINE and XRTPRODUCTS before downloading. Because of the low spatial resolution of XRT $\left(\mathrm{HPD}=18^{\prime \prime}\right.$ at $\left.1.5 \mathrm{keV}\right)$, only one spectrum was extracted for the AGN pair. We used default parameters 


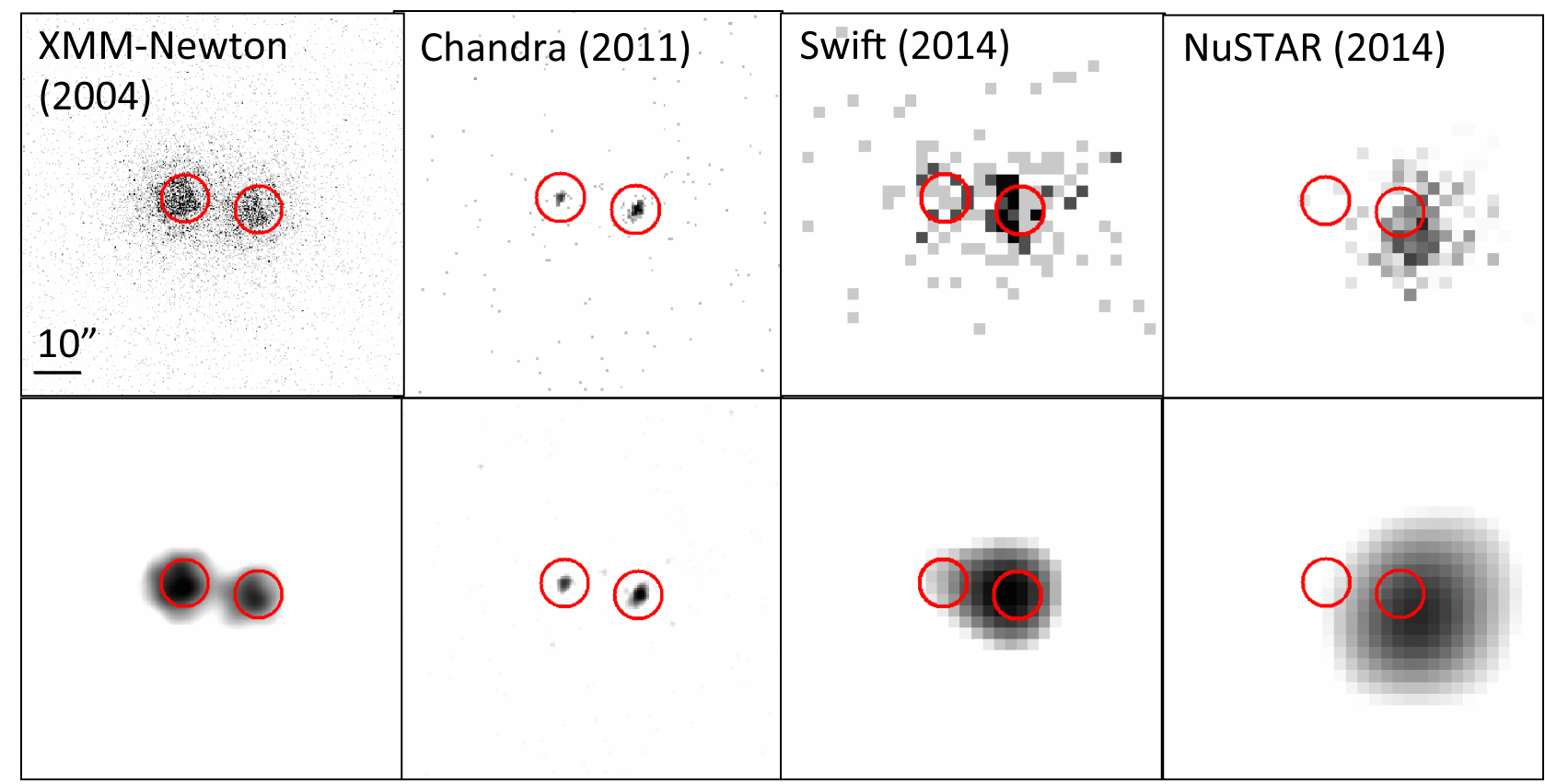

Figure 2. XMM-Newton (0.2-10 keV), Chandra (0.5-8 keV), Swift/XRT (0.5-10 keV), and NuSTAR (3-79 keV) images of ESO 509-IG066 from 2004-2014 showing the progressive fading of the Eastern source (east is left in these images). The red circles mark the positions of the sources and have $5^{\prime \prime}$ radii. All images have the same scale, which is marked on the XMM-Newton image. The top panels show the unsmoothed images and the bottom images show images that have been smoothed with a Gaussian kernel with a radius of $5^{\prime \prime}$ for XMM-Newton, Swift/XRT, and NuSTAR and 2" for Chandra.

Table 1

X-Ray Observations

\begin{tabular}{lrcc}
\hline \hline $\begin{array}{l}\text { Telescope } \\
(1)\end{array}$ & $\begin{array}{c}\text { ObsID } \\
(2)\end{array}$ & $\begin{array}{c}\text { Date } \\
(3)\end{array}$ & $\begin{array}{c}\text { Exposure }(\mathrm{ks}) \\
(4)\end{array}$ \\
\hline XMM-Newton & 0200430801 & 2004 Jan 24 & 13.9 \\
Chandra & 12835 & 2011 Feb 08 & 5.1 \\
NuSTAR & 60061244002 & 2014 Sep 02 & 20.9 \\
Swift/XRT & 00080115002 & 2014 Sep 03 & 6.2 \\
\hline
\end{tabular}

Note. Column (1) gives the telescope name, column (2) lists the observation ID, column (3) gives the start date of the observation, and column (4) gives the exposure time in ks.

(such as extraction radius) while generating the spectrum. The data were then grouped by at least 3 counts per bin. We carry out spectral fitting in the $0.2-7.0 \mathrm{keV}$ energy range where the efficiency of the telescope is highest.

\subsection{Keck}

We obtained observations of the ESO 509-IG066 system with the Keck telescope during 2016. Optical spectroscopy of both nuclei was carried out using the Keck I telescope and the dual-beam Low Resolution Imaging Spectrometer (LRIS, Oke et al. 1995). The $300 \mathrm{~s}$ spectrum, obtained on UT 2016 June 9 in photometric conditions, used the $1 . " 5$ wide slit, the $5600 \AA$ dichroic to split the light, the $600 \ell \mathrm{mm}^{-1}$ grism on the blue $\operatorname{arm}\left(\lambda_{\text {blaze }}=4000 \AA\right)$, and the $600 \ell \mathrm{mm}^{-1}$ grating on the red arm $\left(\lambda_{\text {blaze }}=7500 \AA\right)$. The 1 ." 5 slit corresponds to physical scales of $\sim 1 \mathrm{kpc}$. The observations were obtained at a position angle of $82^{\circ}$ in order to simultaneously observe both galaxies in the system. We processed the data using standard techniques within IRAF, and calibrated the spectrum using standard stars observed using the same instrument configuration on the same night.
In addition to the optical spectroscopy, we acquired near-IR integral-field spectroscopy of the nucleus of the Western galaxy from the adaptive optics (AO)-assisted, near-IR integral-field spectrograph (OSIRIS, Larkin et al. 2006; van Dam et al. 2006; Wizinowich et al. 2006) on the Keck I Telescope taken on UT 2016 April 22. The data were taken in the $K$-band using the $K b b$ filter and the 0 ". 1 pixel scale, resulting in a rectangular field of view of 1 !" $6 \times 6$ !" 4 . The galaxy nucleus was used as a tip-tilt star for the Laser Guide Star AO system. A total of two sky and four on-source exposures of $600 \mathrm{~s}$ each at a position angle of 90 degrees were combined to make the final data cube.

The OSIRIS data were reduced using the OSIRIS data reduction pipeline (ODRP). This performs all the usual steps needed to reduce near-IR spectra, but with the additional routines for reconstructing the data cube. More details can be found in Müller-Sánchez et al. (2016). Flux calibration was performed using an A7V star HD $87035(K=7.5)$.

\subsection{Other Data}

We used WISE (Wright et al. 2010) and NEOWISE-R (Mainzer et al. 2011) data to investigate the IR variability of the galaxy pair, which are spatially resolved by the telescope. ESO 509-IG066 was observed three times by WISE in 2010-2011 and four times by NEOWISE in 2014-2016. Data from the Catalina Sky Survey were used to investigate the variability of the AGN in the optical part of spectrum.

\section{X-Ray Spectral Fitting}

We fit the X-ray data using XSPEC (Arnaud 1996) software version 12.9.0 and used the Cash (Cash 1979) statistic for fitting because of the low number of counts per bin in the Swift data. Both AGNs are modeled in the same way with an absorbed cutoff power law plus pexrav component (Magdziarz \& Zdziarski 1995) simulating scattered radiation from the dusty 


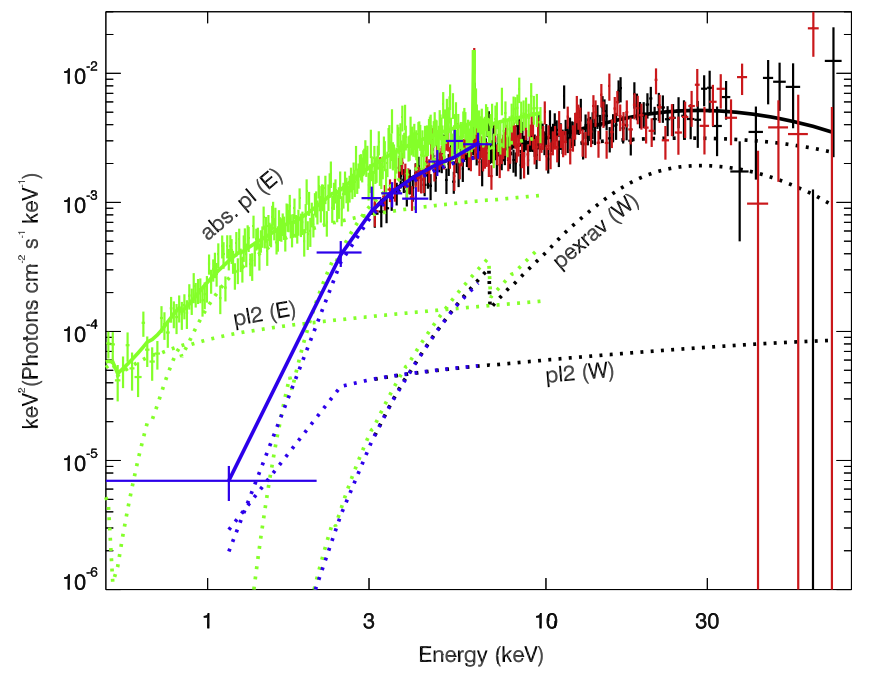

Figure 3. $E F_{\mathrm{E}}$ spectra of the two sources from XMM-Newton (green), NuSTAR (black and red), and Swift/XRT (blue), where both sources have been included in the extraction regions and the spectra have been unfolded through the instrumental responses. The absorbed power-law (abs. pl), secondary powerlaw (p12), and pexrav components used in the fit are marked for each epoch.

torus, plus a narrow iron line at $6.4 \mathrm{keV}$. We take into account Galactic absorption with a wabs model component, the $N_{\mathrm{H}}$ value of which was obtained from the Leiden/Argentine/Bonn survey of Galactic H I (Kalberla et al. 2005), and found to be $6.67 \times 10^{20} \mathrm{~cm}^{-2}$. We also included cross-normalization constants between the X-ray instruments. A secondary power-law component was added, assuming that a fraction of the primary radiation escapes through a patchy absorber without reprocessing, or is scattered into the line of sight. In XSPEC, this model is written: constant*wabs*(constant*cutoffpl + zwabs*cabs (cutoffpl+pexrav +zgauss)). The zwabs*cabs component represents the reprocessing of the X-rays by photo-electric absorption and Compton scattering local to the source. The constant*cutoffpl component represents the secondary power-law component. The pexrav component represents scattered radiation from the torus. All the statistical errors calculated by XSPEC are at the 90 percent confidence level, unless explicitly stated otherwise.

Initially, we extracted a single spectrum for both of the sources from the XMM-Newton, Swift, and NuSTAR data and fitted the three data sets simultaneously. Since G05 showed that the two sources have different spectral properties, specifically the level of absorption, we assumed that we could spectrally decompose the two nuclei in the summed spectra. To do so we used the model described above multiplied by two in order to account for both AGNs within the extraction region. At first we tied the parameters for each source across data sets under the assumption that they did not change between the XMM-Newton observation and the Swift-plus-NuSTAR observations; however, the resulting fit was very poor. A visual inspection of the spectrum revealed that the 2004 XMM-Newton spectrum was significantly different from the $2014 \mathrm{NuSTAR}$ and Swift/XRT spectra, appearing harder (see Figure 3). For this reason, another constant component was applied to the components of one of the sources to account for the possible variability of one source with respect to the other. From this we obtained a very good fit with a C-stat of 556.21 from 589 degrees of freedom (DOF). We found that the constant for one of the sources drops to 0 for the NUSTAR and Swift/XRT data, implying that it is negligible in the 2014 data. The results of this fit are summarized in Table 2. We identify the source with the highest $N_{\mathrm{H}}$ value as the Western source and the other as the Eastern source, since these match the parameters from G05, who carried out spatially resolved analysis on the galaxies.

The first constant in the spectral model is used to account for differences between instruments and possible variability effects. We fix it to 1 for NuSTAR FPMA and let it float for all other instruments. Constants obtained by fitting the spectrum simultaneously are: $1.01 \pm 0.04$ for FPMB, $1.26_{-0.31}^{+0.22}$ for XMM-Newton-pn, and $1.01_{-0.13}^{+0.15}$ for Swift/XRT. The cross-normalization for XMM-Newton is higher, possibly due to variability between 2004 and 2014. However, it is still consistent with unity within the 90 percent uncertainties. Noticing this, we investigated the variability of the Western source. We tried freeing the photon index and column densities of this source in XMM-Newton data, but the resulting values stayed constant within the uncertainties. The other crossnormalization constants are consistent with calibrated values from Madsen et al. (2015).

To investigate the variability of the two sources individually, we analyzed the Chandra observation from 2011. For XMMNewton, while the $16^{\prime \prime}$ separation of the sources is similar to the FWHM of the telescope's PSF, we extracted the spectra of each source using small $7^{\prime \prime}-8^{\prime \prime}$ radius apertures following G05, bearing in mind that each spectrum will be contaminated by the other's PSF wings. First, we extracted XMM-Newton and Chandra spectra of the Western source only. We simultaneously fit the spectra using a simple spectral model in the form constant*wabs (constant*po + zwabs*cabs (po + zgauss)). The power law did not require a cutoff and the pexrav component was not required since these features are not significant in the soft X-ray range (below $10 \mathrm{keV}$ ). As the Chandra observation is only $5 \mathrm{ks}$, we were not able to constrain all the parameters of the spectral model. We achieved a very good fit of 69.49/92 C-stat/DOF when freeing $f_{\mathrm{pl} 2}$ (the fraction of the secondary power-law to the primary one), $N_{\mathrm{H}}$, and the cross-normalization constant, and fixing all other parameters. The results of the fit are shown in Table 3 and in Figure 4. We notice an increase in the $N_{\mathrm{H}}$ of the absorber from $6 \pm 1 \times 10^{22} \mathrm{~cm}^{-2}$ to $1.2 \pm 0.2 \times 10^{23} \mathrm{~cm}^{-2}$, though the uncertainties increased as well. The equivalent width of the $\mathrm{Fe}$ $\mathrm{K} \alpha$ line is $82_{-78}^{+95} \mathrm{eV}$ during the XMM-Newton observation and $68_{-68}^{+162} \mathrm{eV}$ during the Chandra observation. The cross-normalization constant between the measurements is consistent with unity at the 90 percent confidence level.

We extracted the spectrum of the Eastern source from XMMNewton and Chandra observations as done with the Western source. The source is detected in both observations, but the flux from the 2011 Chandra observation is much lower than that from the 2004 XMM-Newton observation. We fit the spectrum simultaneously using the same model used for the Western source. In this case, the zgauss component is not significant anymore and again the pexrav component and high-energy cutoff were not required. We obtained a good fit with 85.18/ $115 \mathrm{C}$-stat/DOF by tying all the parameters for Chandra to the $X M M-N e w t o n$ parameters except for the cross-normalization constant, which now accounts mostly for the drop in flux of the object. Upon freeing the other Chandra spectral parameters, the fit becomes unconstrained. The results are summarized in Table 3 and Figure 5. We find that the normalization of the 
Table 2

Results of Simultaneous Fitting of Both Sources Using NuSTAR, Swift, and XMM-Newton Data

\begin{tabular}{lrrrcrr}
\hline $\begin{array}{l}\text { Source } \\
(1)\end{array}$ & $\begin{array}{c}N_{\mathrm{H}} \\
(2)\end{array}$ & $\begin{array}{c}\Gamma \\
(3)\end{array}$ & $\begin{array}{c}E_{\mathrm{C}} \\
(4)\end{array}$ & $\begin{array}{c}\text { Power-law Norm. } \\
(5)\end{array}$ & $\begin{array}{c}f_{\mathrm{pl} 2} \\
(6)\end{array}$ & $\begin{array}{c}\text { Pexrav Norm. } \\
(7)\end{array}$ \\
\hline West & $7.3_{-1.4}^{+1.5}$ & $1.65_{-0.20}^{+0.23}$ & $71_{-30}^{+150}$ & $1.56_{-0.52}^{+0.68} \times 10^{-3}$ & $6_{-l}^{+25} \times 10^{-3}$ & $1.5_{-1.2}^{+2.8} \times 10^{-3}$ \\
East & $0.60_{-0.15}^{+0.22}$ & $1.84_{-0.32}^{+0.62}$ & 500 & $5.9_{-1.2}^{+1.5} \times 10^{-4}$ & $0.11_{-l}^{+u}$ & $\ldots$ \\
\hline
\end{tabular}

Note. Column (1) gives source name, column (2) gives the $N_{\mathrm{H}}$ value in units of $10^{22} \mathrm{~cm}^{-2}$, column (3) gives the photon index of the cutoff power law, and column (4) shows the exponential cutoff energy of the cutoff power law in keV. Column (5) lists the normalization of the power law in units of photons keV ${ }^{-1} \mathrm{~cm}^{-2} \mathrm{~s}^{-1}$ at $1 \mathrm{keV}$, column (6) gives the fraction of the leaked power-law model to the primary one (" $-l$ " indicates that this fraction is unconstrained at the lower end, and " $+\mathrm{u}$ " indicates that the fraction is unconstrained at the upper end), column (7) shows the normalization of the pexrav component in units of photons keV $\mathrm{cm}^{-2} \mathrm{~s}^{-1}$ at $1 \mathrm{keV}^{-}$and column (8) lists the normalization of a Gaussian component representing the iron line at $6.4 \mathrm{keV}$ in units of total photons cm $\mathrm{c}^{-2} \mathrm{~s}^{-1}$ in the line. The equivalent width of this line is $150 \mathrm{eV}$.

Eastern source decreased by a factor of $\sim 10$ between 2004 , when XMM-Newton observed it and 2011, the date of the Chandra observation.

In the final part of X-ray analysis, we focus on fluxes from both of the sources and their changes over time. We calculate the flux using cflux, which provides the observed flux of the combined spectral models. We calculate the flux in two different bands: soft (0.5-2.0 keV) and hard (2.0-8.0 keV). From the Chandra and XMM-Newton data, we obtain fluxes of the sources separately, while for the Swift-plus-NuSTAR data, the calculated flux is the sum of both sources. However, we list it as the flux of Western source only, since the Eastern source is undetected. We include the upper limit at the 90 percent confidence level for the Eastern source flux calculated using spectral modeling. We plot the results from this analysis in Figure 6.

The system was reported by Swift/BAT as having 14-195 keV flux of $1.4 \times 10^{-11} \mathrm{erg} \mathrm{cm}^{-2} \mathrm{~s}^{-1}$ (Cusumano et al. 2010; Baumgartner et al. 2013), which is an average over the period 2005-2009. We estimate the 14-195 keV flux of ESO 509-IG066 from the 2014 NuSTAR observation by extrapolating our spectral model up to $195 \mathrm{keV}$. We find that the flux during the NuSTAR observation is $1.5 \times 10^{-11}$ erg cm $\mathrm{cm}^{-2} \mathrm{~s}^{-1}$, which is consistent with the Swift/BAT flux reported, implying that there is no evidence for a drop in X-ray flux in this band. However, this is not surprising, since the Western source, which has remained relatively constant, dominates at high energies.

\section{Long-term Optical and IR Light Curves}

To investigate the cause of the X-ray variability of the Eastern source, we examined the optical and IR light curves. We used the Catalina Real-Time Transient Survey (CRTS) to determine the $V$-band optical brightness of the Eastern source over time. The measurements were taken from 2005 August until 2013 July, so the survey covers a large time period between our X-ray observations.

We then analyzed the mid-IR brightness of both sources using WISE and NEOWISE data as described in Section 2. The sources were observed during seven epochs, three times in 2010-2011 and four times in 2014-2016. The reduced $\chi^{2}$ of the standard profile fitting technique for WISE point sources listed in the AllWISE catalog indicates that the sources are extended. For this reason, we use small-aperture photometry, extracted from regions of 5.15 also listed in the AllWISE catalog. Lastly, since the W3 and W4 passbands are not available for NEOWISE, we do not include them in our analysis. We present this photometry in Table 4.

First, we note that the mid-IR colors of the Eastern galaxy are relatively blue, with $\mathrm{W} 1-\mathrm{W} 2 \simeq 0.3$, which indicates that the bands are dominated by stellar emission. For the Western galaxy $\mathrm{W} 1-\mathrm{W} 2 \simeq 0.7$, which is more consistent with being dominated by the AGNs (Stern et al. 2012). Therefore, any drop in mid-IR flux from the AGNs in the Eastern galaxy will probably be washed out by the host galaxy. While the flux in W1 from the Eastern galaxy does shows a 20\% drop during the 2010-2011 WISE observations and the AllWISE catalog gives it the maximum probability that the flux was not constant with time, the drop in flux is not sustained, as seen in the 2014-2016 NEOWISE data, which show a recovery of the initial flux. Finally, the expected optical-to-K-band time lag using our assumed cosmology is 25 days, a much shorter timescale than the cadence of the WISE data (although the W1, W2 emission regions may be slightly larger than the K-band emission region).

We convert the $V$-band magnitude from CRTS and the WISE $\mathrm{W} 1$ and $\mathrm{W} 2$ magnitudes to $\nu F_{\nu}$ fluxes in order to compare to the $\mathrm{X}$-ray data. We plot this multiband light curve in Figure 6. While the X-ray flux from the Eastern galaxy has dropped by a factor of 10 in the X-ray bands, the flux at optical and mid-IR wavelengths has remained relatively constant over the long base line. No major variations are seen in the flux from the Western galaxy at X-ray, optical, or mid-IR wavelengths.

\section{Optical and IR Spectroscopy}

In order to gain further insight into the nature of the interaction between the galaxies and the drop in X-ray flux from the Eastern nucleus, we analyzed the optical spectra of the two nuclei. The processed Keck/LRIS spectra are shown in Figure 7. We use the penalised PiXel Fitting software (pPXF, Cappellari \& Emsellem 2004) to measure stellar kinematics and the central stellar velocity dispersion with the Indo-U.S. CaT, and MILES empirical stellar library (3465-9468 $\AA$ Vazdekis et al. 2012). We fit the residual spectra for emission lines after subtracting the stellar templates with the PYSPECKIT software following Berney et al. (2015) and correct the narrow line ratios $(\mathrm{H} \alpha / \mathrm{H} \beta)$, assuming an intrinsic ratio of $R=3.1$ and the Cardelli et al. (1989) reddening curve. In the case of a $\mathrm{H} \beta$ nondetection, we assume the $3 \sigma$ upper limits for the extinction correction.

The optical spectrum of the Western galaxy exhibits strong forbidden transition lines from [O III] and [N II] and BPT 
Table 3

Simultaneous Fitting Results of Both Sources Using XMM-Newton and Chandra Data

\begin{tabular}{|c|c|c|c|c|c|c|}
\hline $\begin{array}{l}\text { Source } \\
\text { (1) }\end{array}$ & $\begin{array}{l}N_{\mathrm{H}} \\
(2)\end{array}$ & $\begin{array}{c}\Gamma \\
(3)\end{array}$ & $\begin{array}{c}\text { Power-law Norm. } \\
\text { (4) }\end{array}$ & $\begin{array}{l}f_{\mathrm{pl} 2} \\
(5)\end{array}$ & $\begin{array}{l}\text { Iron Line Norm. } \\
(6)\end{array}$ & $\begin{array}{c}\text { Cross-normalization Constant } \\
\text { (7) }\end{array}$ \\
\hline \multicolumn{7}{|c|}{ Western Source } \\
\hline \multicolumn{7}{|c|}{ Eastern Source } \\
\hline
\end{tabular}

Note. Column (1) lists the instrument that measured the data, column (2) gives the $N_{\mathrm{H}}$ value in units of $10^{22} \mathrm{~cm}^{-2}$, column (3) gives the photon index of the power law, column (4) lists the normalization of the power law in units of photons $\mathrm{keV}^{-1} \mathrm{~cm}^{-2} \mathrm{~s}^{-1}$ at $1 \mathrm{keV}$, column (5) gives the fraction of the secondary power law to the primary one, column (6) gives the normalization of the zgauss component in units of total photons $\mathrm{cm}^{-2} \mathrm{~s}^{-1}$ in the line, and column (7) shows the crossnormalization constant between the two measurements.

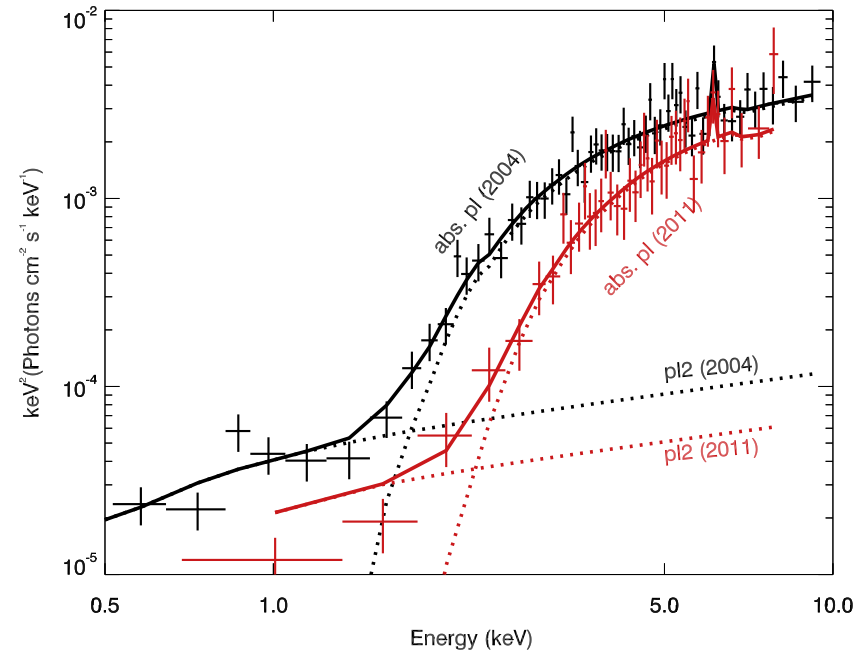

Figure 4. $E F_{\mathrm{E}}$ spectra of the Western source from XMM-Newton (black) and Chandra (red) where the spectra have been unfolded through the instrumental responses. The absorbed power-law (abs. pl) and secondary power-law (pl2) components used in the fit are marked for each epoch.

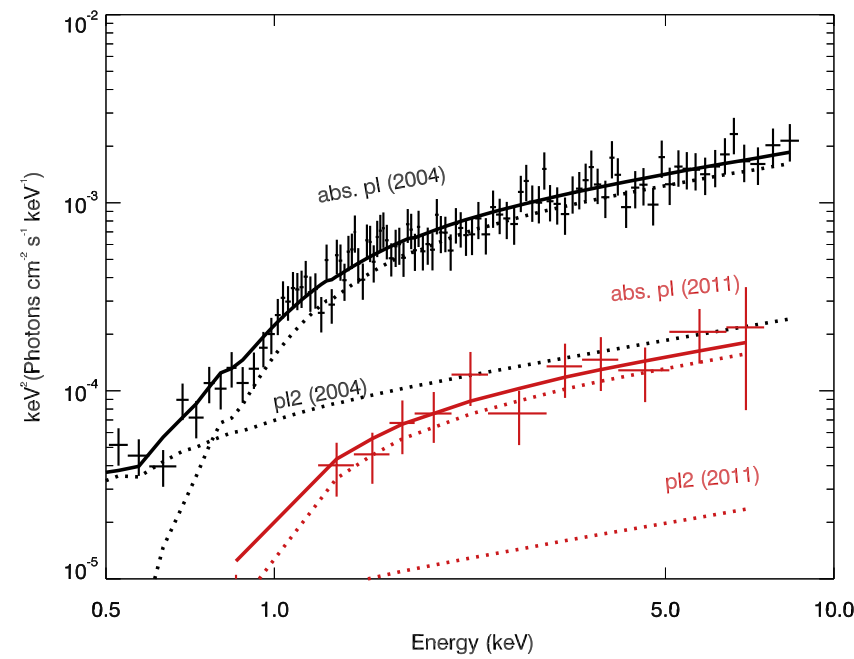

Figure 5. $E F_{\mathrm{E}}$ spectra of the Eastern source from XMM-Newton (black) and Chandra (red) where the spectra have been unfolded through the instrumental responses. The absorbed power-law (abs. pl) and secondary power-law (pl2) components used in the fit are marked for each epoch.
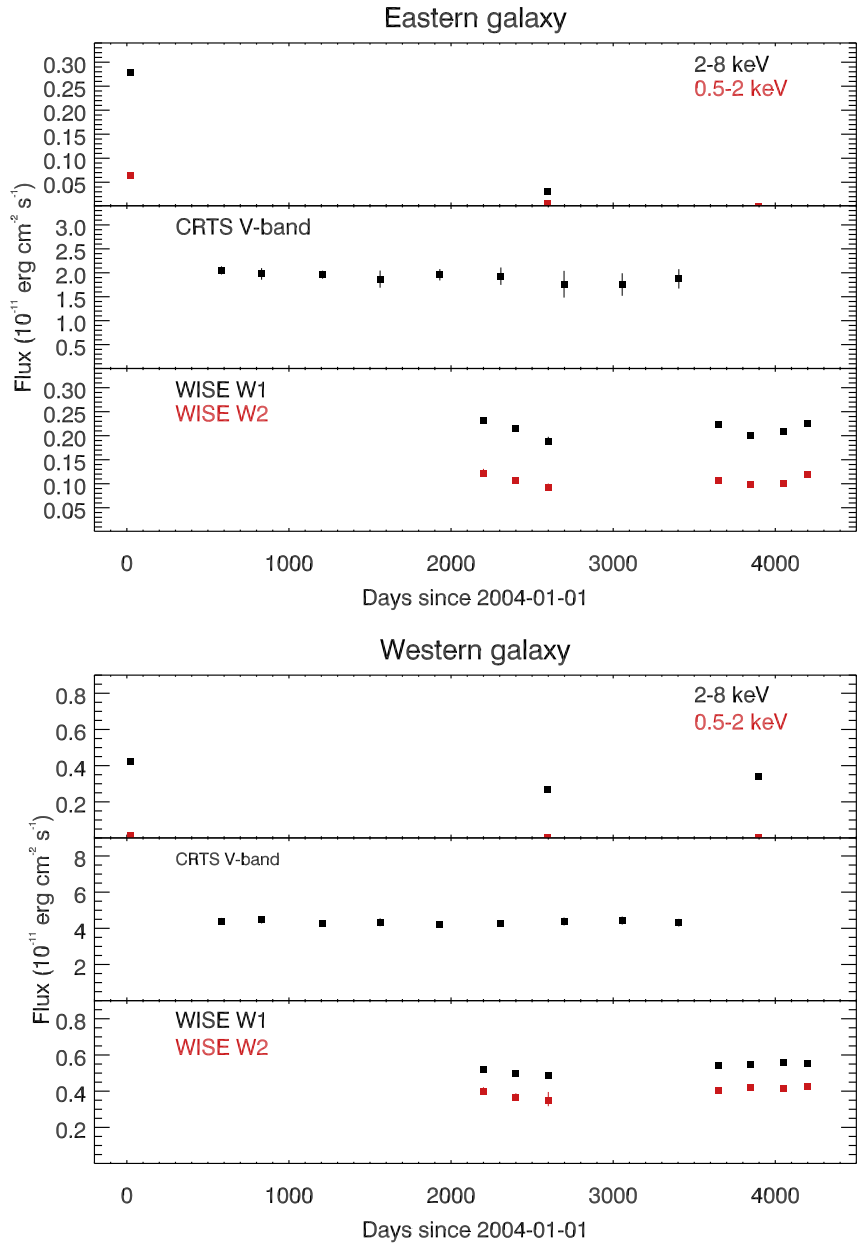

Figure 6. Multiband light curves of both the Eastern (top) and Western (bottom) galaxies, covering the period 2004-2014. While the X-ray flux from the Eastern galaxy has dropped by a factor of 10 in the X-ray bands, the flux at optical and mid-IR wavelengths has remained constant. No major variations are seen in the flux from the Western galaxy at X-ray, optical, or mid-IR wavelengths.

diagnostics confirm that the galaxy hosts a Seyfert 2 nucleus (Figure 8). The Balmer-decrement-corrected [O III] flux is $1.91 \times 10^{-13} \mathrm{erg} \mathrm{cm}^{-2} \mathrm{~s}^{-1}$. We measure a velocity dispersion of $118 \pm 37 \mathrm{~km} \mathrm{~s}^{-1}$ in the $\mathrm{CaH}+\mathrm{K}$ and $\mathrm{Mgb}$ region and 
Table 4

WISE and NEOWISE Photometry of Both Galaxies

\begin{tabular}{|c|c|c|c|c|}
\hline \multirow[b]{2}{*}{ (1) } & \multicolumn{2}{|c|}{ Eastern Source } & \multicolumn{2}{|c|}{ Western Source } \\
\hline & $\begin{array}{l}\text { W1 } \\
\text { (2) }\end{array}$ & $\begin{array}{l}\text { W2 } \\
\text { (3) }\end{array}$ & $\begin{array}{l}\text { W1 } \\
\text { (4) }\end{array}$ & $\begin{array}{l}\text { W2 } \\
\text { (5) }\end{array}$ \\
\hline AllWISE 2 & $12.75 \pm 0.02$ & $12.52 \pm 0.01$ & $11.83 \pm 0.02$ & $11.19 \pm 0.04$ \\
\hline AllWISE 3 & $12.89 \pm 0.04$ & $12.68 \pm 0.06$ & $11.86 \pm 0.03$ & $11.25 \pm 0.12$ \\
\hline NEOWISE-R 1 & $12.71 \pm 0.02$ & $12.53 \pm 0.03$ & $11.75 \pm 0.08$ & $11.07 \pm 0.03$ \\
\hline NEOWISE-R 2 & $12.83 \pm 0.03$ & $12.62 \pm 0.05$ & $11.73 \pm 0.02$ & $11.04 \pm 0.04$ \\
\hline
\end{tabular}

Note. Column (1) shows the observational epoch and columns (2)-(5) list the WISE and NEOWISE magnitudes (Vega) of both galaxies in the W1 and W2 bands.
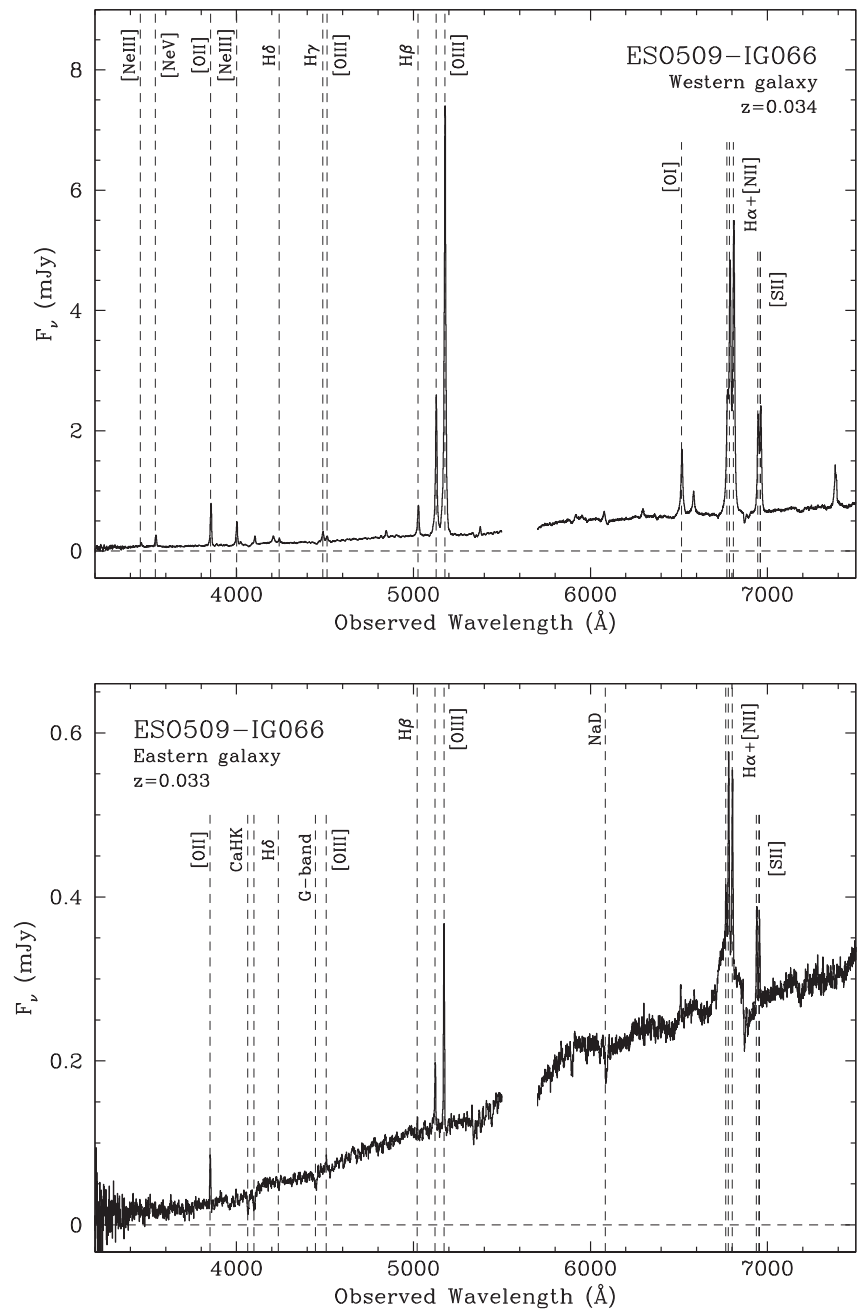

Figure 7. Keck/LRIS optical spectra of the nucleus of the Western galaxy (top) showing strong forbidden lines typical of a Seyfert 2 and the Eastern galaxy (bottom), which reveals a broad $\mathrm{H} \alpha$ beneath narrow $\mathrm{H} \alpha+[\mathrm{N}$ II] lines. This is the first reported detection of broad optical lines from this galaxy and indicates that the nucleus is not strongly absorbed, arguing against heavy absorption behind the drop in X-ray flux.

$124 \pm 27 \mathrm{~km} \mathrm{~s}^{-1}$ in the Calcium triplet absorption lines. We show the fit to the Calcium triplet lines in Figure 9.

In the LRIS spectrum of the Eastern galaxy a broad $\mathrm{H} \alpha$ line is detected with a width of $4226 \mathrm{~km} \mathrm{~s}^{-1}$ characteristic of a Seyfert 1 nucleus (Figure 10); however, the $\mathrm{H} \beta$ line is very weak, and so would be classified as a Seyfert 1.9
(Osterbrock 1981). Using an upper limit to the flux of the narrow $\mathrm{H} \beta$ line, we find that the BPT diagnostics also confirm the presence of a Seyfert nucleus in this source (Figure 8). The Eastern galaxy has a velocity dispersion that is consistent with the instrumental resolution $\left(<100 \mathrm{~km} \mathrm{~s}^{-1}\right)$ in the $\mathrm{CaH}+\mathrm{K}$ and $\mathrm{Mgb}$ region. However, due to the broad $\mathrm{H} \alpha$ line, the Calcium triplet absorption lines are likely contaminated by AGN emission.

Sekiguchi \& Wolstencroft (1992) presented optical spectroscopic observations of the two galaxies, taken with the $1.9 \mathrm{~m}$ South African Astronomical Observatory, also finding the Western nucleus to be a Seyfert 2. They, however, classified the Eastern nucleus as a LINER or H II galaxy. It is unclear if the broad $\mathrm{H} \alpha$ line was undetected in their observations or not present at that time when they were made, between 1987 and 1990. Our new detection of broad $\mathrm{H} \alpha$ strongly suggests that our view of the Eastern nucleus is largely unobscured. Some reddening may be present in order to explain the non-detection of a broad $\mathrm{H} \beta$ line. We can estimate the amount of reddening from the flux ratio of the $\mathrm{H} \alpha$ and $\mathrm{H} \beta$ lines, known as the Balmer decrement. Given the upper limit on the flux of the $\mathrm{H} \beta$ line, the lower limit on the Balmer decrement is 4.7. Assuming an intrinsic value of 3.1, this corresponds to a lower limit on the reddening of $E(B-V)=0.36$, which, assuming the Galactic gas-to-dust ratio corresponds to $N_{\mathrm{H}} \sim 10^{21} \mathrm{~cm}^{-2}$. In order to suppress the X-ray flux from the Eastern nucleus such that it is not detected by NuSTAR, the obscuration must be at least 4 orders of magnitude higher, around $10^{25} \mathrm{~cm}^{-2}$. If this were the case, the implied reddening in the optical means that the broad $\mathrm{H} \alpha$ line would not be detectable. A possibility remains, however, that the broad $\mathrm{H} \alpha$ line results from scattered light from the nucleus that would not be subjected to the heavy lineof-sight absorption that the X-rays may be subjected to.

In addition to the optical spectroscopy of the two nuclei, we also acquired near-IR $K$-band integral-field spectroscopy of the inner $\sim \mathrm{kpc}$ of both nuclei. The integrated spectrum of the Western nucleus (Figure 11) reveals several transitions of molecular hydrogen, $\operatorname{Br} \gamma$ and $\operatorname{Br} \delta$ from atomic hydrogen, as well as transitions from ionized gas ([Si VI] and [He I]). The molecular hydrogen transitions indicate the presence of large amounts of molecular gas, while the emission from highly ionized gas confirms the presence of a powerful AGN. The integrated spectrum of the Eastern nucleus is featureless, however, with no emission lines detected.

We use the strong $\mathrm{H}_{2} 1-0 \mathrm{~S}(1)$ emission line at $2.12 \mu \mathrm{m}$ to map the velocity of the gas within the inner $\sim \mathrm{kpc}$ of the Western galaxy, fitting it with a single Gaussian for each pixel 

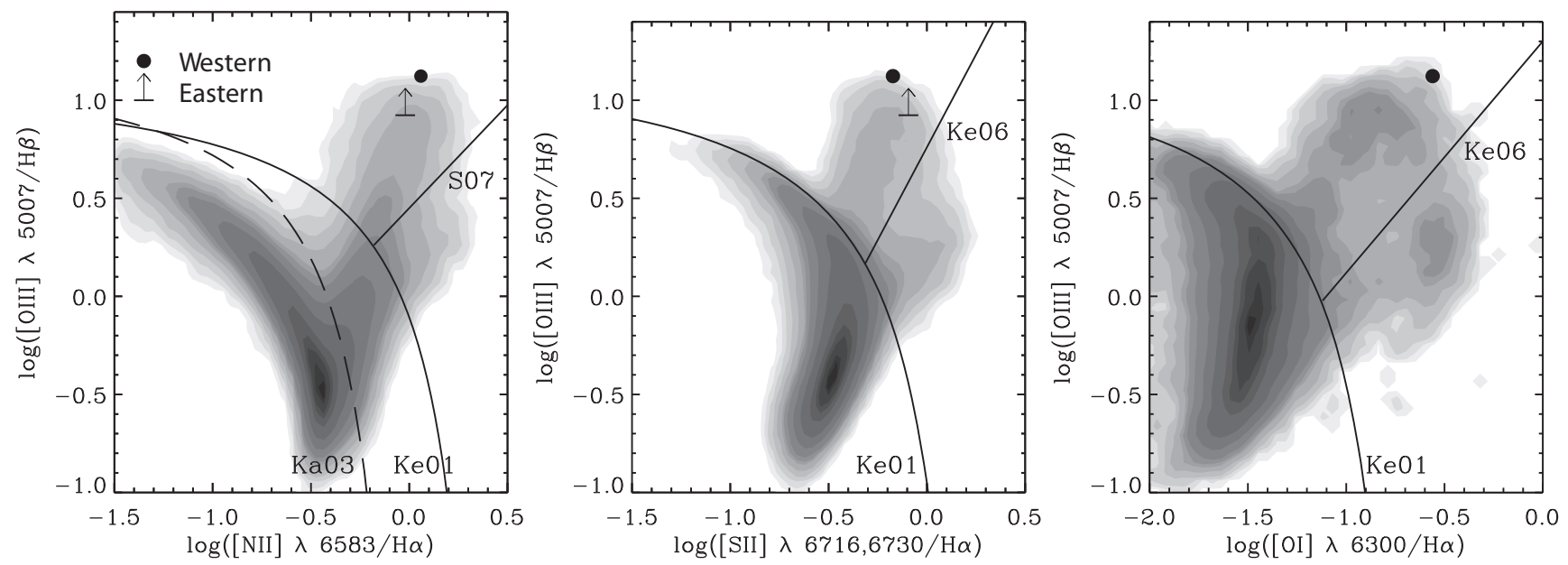

Figure 8. BPT narrow emission-line diagnostic diagrams for the Western (black dot) and Eastern (black bar) nuclei. The solid black curve shows the separation between star-forming galaxies, which lie below the curve, and AGNs, which lie above the curve, from Kewley et al. (2001). The dashed curved line shows the same separation, but from Kauffmann et al. (2003). The solid straight line shows the separation between Seyferts, which fall left of the line, and LINERs, which fall to the right of the line, from Kewley et al. (2006). The Western nucleus is in the Seyfert section in all three diagrams. The Eastern nucleus only has an upper limit on the H $\beta$ flux, but the corresponding lower limit of the ratio is in the Seyfert region, thus both galaxies are classified as Seyferts from our data.

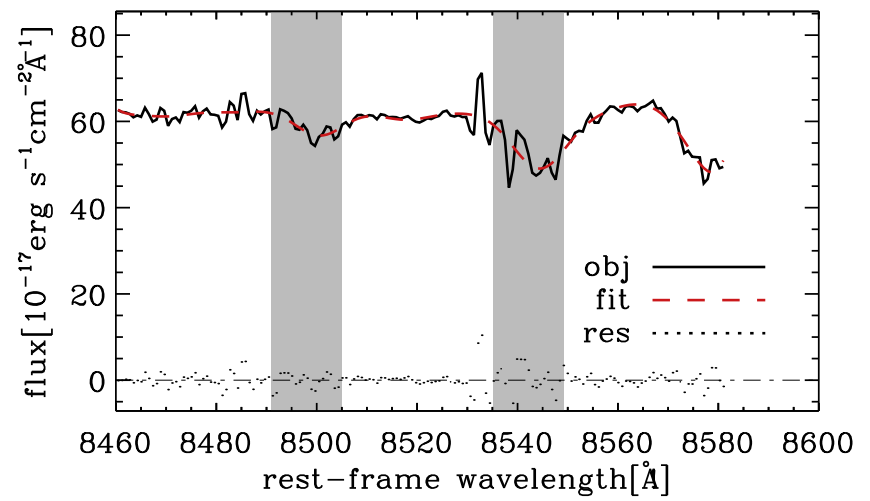

Figure 9. Zoom-in on the Keck/LRIS optical spectrum of the Western galaxy in the region where the Calcium triplet absorption occurs, which we use to measure the velocity dispersion of the stars.

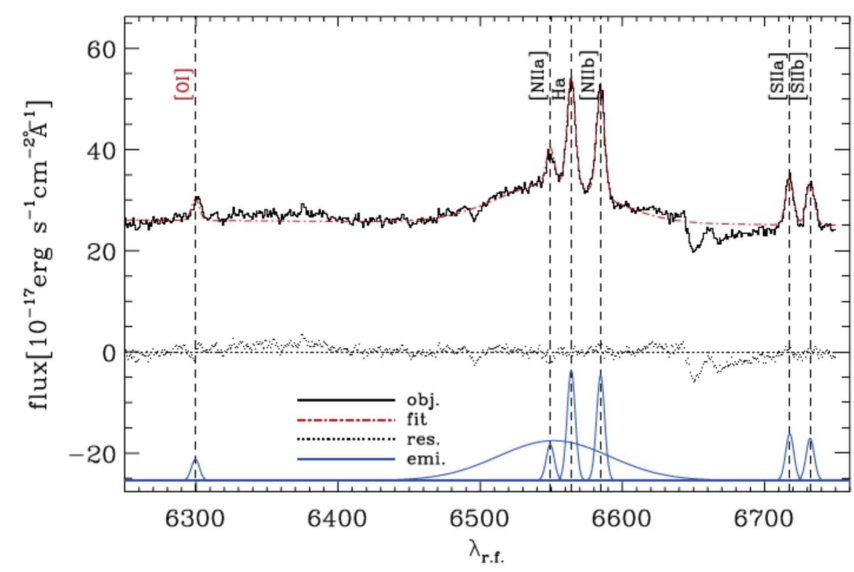

Figure 10. Zoom-in on the Keck/LRIS optical spectrum of the Eastern galaxy in the region where the broad $\mathrm{H} \alpha$ line was detected, showing the spectral decomposition.

in the field of view. Figure 12 presents the flux, velocity, and the velocity dispersion inferred from these measurements. We find that within the inner 200-300 pc of the galaxy, the gas rotates in an ordered fashion, with a systematic velocity toward us to the west of the nucleus, and a systematic velocity away

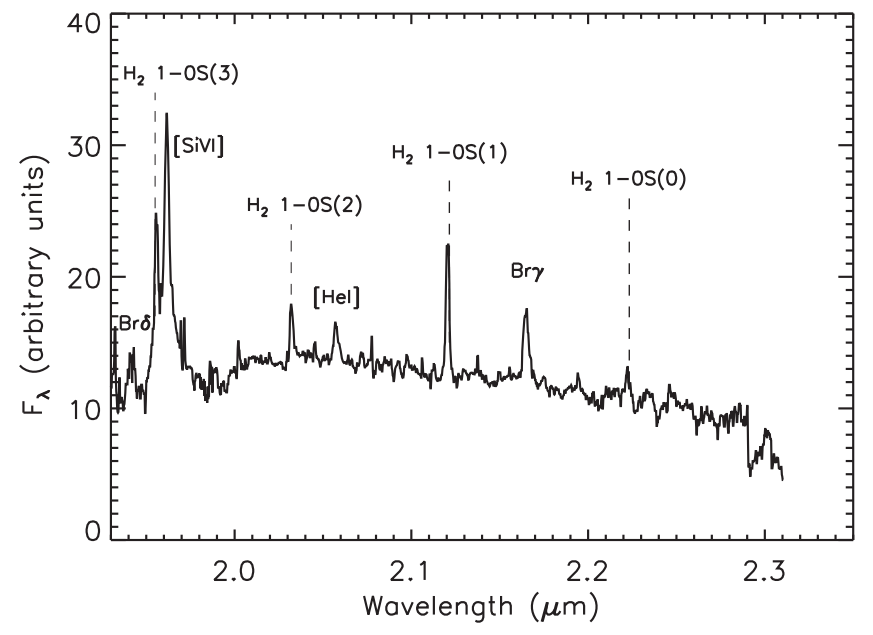

Figure 11. Integrated Keck/OSIRIS spectrum of the Western nucleus in the $K$-band (rest frame). The individual spectra were added over an aperture of 0 ". 6 diameter centered at the peak of continuum emission in the near-IR. Several transitions of molecular hydrogen can be seen, where the $2.12 \mu \mathrm{m} \mathrm{H}_{2} 1-0 \mathrm{~S}(1)$ emission line is the strongest. Ionized gas emission is also detected in this galaxy ([Si VI], $\mathrm{Br} \delta, \quad \mathrm{Br} \gamma$, and $[\mathrm{He} \mathrm{I}])$, confirming the presence of a powerful AGN.

from us to the east of the nucleus. The velocity dispersion is also low $\left(<100 \mathrm{~km} \mathrm{~s}^{-1}\right)$. However, at $\sim 1 \mathrm{kpc}$ to the east of the nucleus, a region of gas appears to have motion that is redshifted toward us, opposite to the direction of ordered rotation in that region. This region also shows very high velocity dispersion of $455 \mathrm{~km} \mathrm{~s}^{-1}$ (typical error $10-20 \mathrm{~km} \mathrm{~s}^{-1}$ ). A high dispersion is an indication of shocks and perturbed kinematics. These usually correspond to outflows, but they are also associated with inflows, particularly from merger processes (Medling et al. 2015; Müller-Sánchez et al. 2016). We have mapped the gas outflow from the Western AGN using the high-ionization [Si VI] line, which shows a different morphology from the molecular gas, orientated in the north-south direction. We therefore find it unlikely that the perturbed molecular gas in the east is caused by an AGN outflow. Since this galaxy appears to be interacting with its neighbor to the east, we interpret these observations as signatures of an inflow 

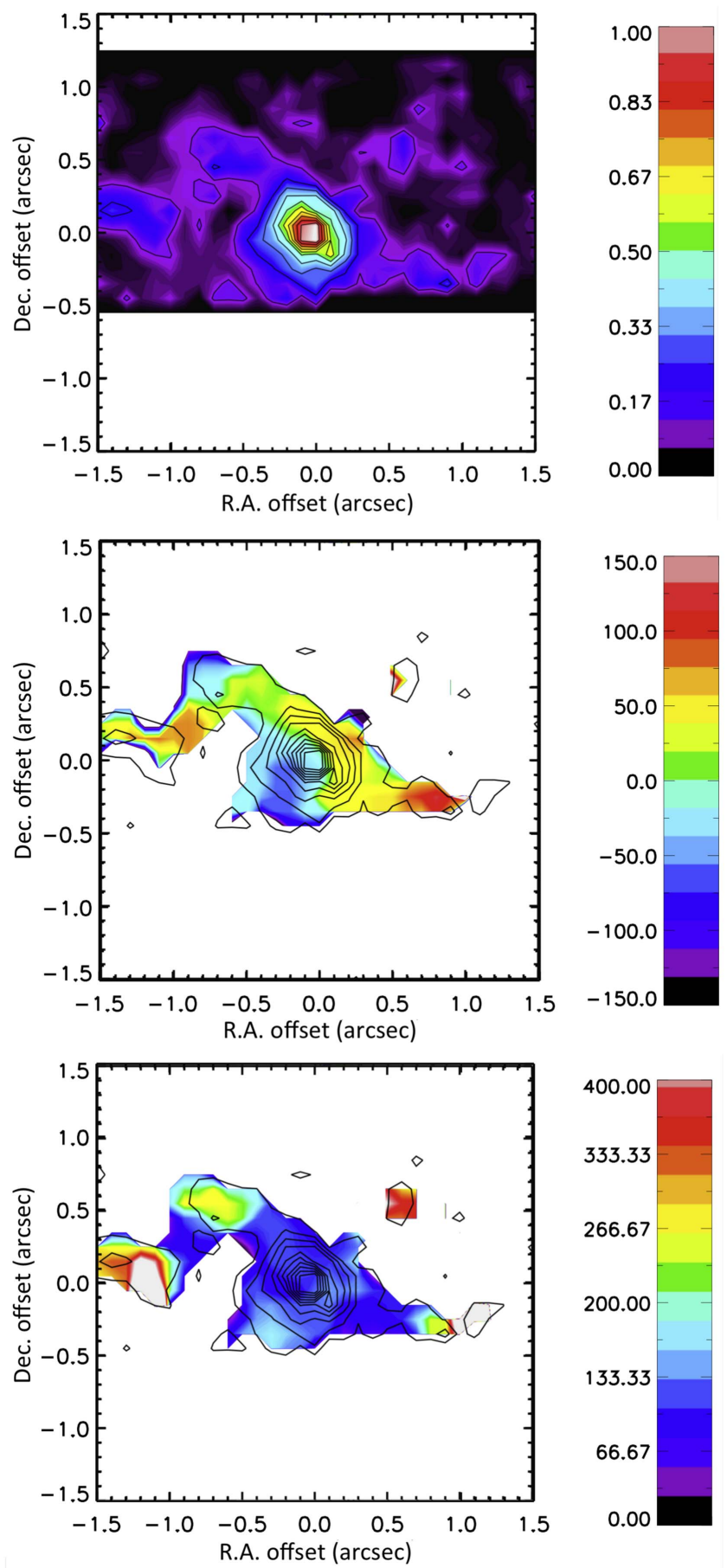

Figure 12. Maps of the $\mathrm{H}_{2} 1-0 \mathrm{~S}$ (1) flux (top), velocity (middle), and velocity dispersion (bottom) in the nucleus of the Western galaxy acquired from Keck/ OSIRIS near-IR integral-field spectroscopy observations. The color scale is in $\mathrm{km} \mathrm{s}^{-1}$ and the angular scale is $700 \mathrm{pc} /{ }^{\prime \prime}$ at the redshift of the system and our assumed cosmology. The central 200-300 pc shows ordered rotation (PA of the kinematic major axis $\sim 135^{\circ}$ ), whereas the gas at $\sim 1 \mathrm{kpc}$ to the east of the nucleus appears to be systematically redshifted (top). The same region of gas also shows a high (455 $\mathrm{km} \mathrm{s}^{-1}$ ) velocity dispersion, pointing toward a physical interaction between the galaxies (bottom). The contours delineate the molecular gas morphology and are normalized to the peak of emission. Each contour represents a change in flux of $10 \%$.

of gas caused by a physical interaction between the galaxies. While a region of high velocity dispersion is also seen to the northwest of the nucleus, the signal-to-noise is low and has significantly lower velocity dispersion $\left(300 \mathrm{~km} \mathrm{~s}^{-1}\right)$ than the region to the east. Furthermore, there is no evidence that the velocity is systematically different from the ordered rotation seen in the rest of the nucleus.

Therefore, we conclude that while the nuclei are separated by $\sim 11 \mathrm{kpc}$, the effect of the interaction is seen on the gas within the inner $\sim 1 \mathrm{kpc}$ of the Western galaxy. This clearly shows that galaxy interactions like the one in ESO 509-IG066 can have a significant impact on the motion of gas within the nuclei and the feeding of the central SMBH.

\section{Discussion}

One of the notable features of the X-ray observations spanning 12 years is the drop, by a factor of 10 , of the flux of the Eastern source. There are two possible straightforward explanations for this observed drop in X-ray flux. The decrease in flux could be caused by an increase in the column density of the absorber. A cloud of gas and dust might be passing in front of the nucleus along the line of sight obscuring the source (e.g., NGC 1365, Risaliti et al. 2009; Rivers et al. 2015b). Provided the column density is extremely high (above $10^{25} \mathrm{~cm}^{-2}$ ), the only received X-ray radiation would be that escaping through gaps between clouds, assuming that the covering fraction is not $100 \%$, or light that has been scattered into our line of sight. The spectrum would then resemble results obtained from the 2011 Chandra observation, being lower in flux with approximately the same spectral shape. An extreme $N_{\mathrm{H}}$ would be required so that even emission above $10 \mathrm{keV}$ is suppressed by Compton scattering since the Eastern source is not visible in the NuSTAR image (Figure 2). With an angular separation of $16^{\prime \prime}$, the nuclei are far enough apart to be distinguishable with NUSTAR, whose PSF has a 18" FWHM (Harrison et al. 2013; Madsen et al. 2015). The X-ray emission in the NuSTAR image peaks strongly at the position of the Western source, with no indication for the Eastern one. Furthermore, there is no evidence for $\mathrm{Fe}-\mathrm{K} \alpha$ emission in the Chandra spectrum of the source. In AGNs, significant obscuration is usually but not always associated with $\mathrm{Fe}-\mathrm{K} \alpha$ fluorescence emission. The absence of an Fe-K line suggests absorption is not responsible for the flux decrease.

The second possibility is that the intrinsic X-ray luminosity of the AGN itself decreased by a factor of at least 10 over the past 10 years, due to a decrease in coronal activity, which could have been caused by a drop in the mass accretion rate. This would explain the spectral shape seen in the 2011 Chandra observation, which is fitted well by a model with similar physical parameters, such as column density and power-law slope. Assuming a similar luminosity for the nucleus in 2014 and 2011, this scenario also agrees with the non-detection of the AGN by NuSTAR and its very faint detection by Swift/ XRT. The Eastern nucleus was weak in the hard X-ray band in 2004 with respect to the Western source, and thus would be undetectable by NUSTAR above $3 \mathrm{keV}$ after a decrease by a factor of 10. Additional evidence in favor of the drop in accretion rate comes from the optical spectrum, which reveals a weak but significantly detected broad $\mathrm{H} \alpha$ line from the nucleus of the Eastern galaxy, which must come from close to the central engine. If the dimming were due to obscuration, it would require an extremely low dust-to-gas ratio for the X-ray flux to have undergone such suppression, while the $\mathrm{H} \alpha$ line remains visible, although the possibility still exists that the $\mathrm{H} \alpha$ line may be scattered light. 
However, no emission lines were detected in the NIR from the Eastern nucleus. This implies that there are not enough ionizing photons to produce emission lines of ionized gas in the near-IR (like $\mathrm{Br}-\gamma$, [He I], or [Si VI] seen from the Western nucleus). Also, the lack of molecular hydrogen indicates that there is not sufficient gas to maintain the active nucleus. This is consistent with our interpretation that the accretion rate of the eastern nucleus has dropped.

We discuss the scenario that the merging of the two galaxies is directly linked to the change in accretion rate of the Eastern AGN. First, galaxy merger simulations predict large fluctuations in black hole accretion rate during the final stages of a merger (e.g., Van Wassenhove et al. 2012; Gabor et al. 2016). Although the time resolution of most simulations $\left(\sim 10^{3}\right.$ years $)$ is much longer than our observational timescale, results on much shorter timescales $(\sim 10$ years $)$ also reveal similar accretion rate fluctuations (J. Gabor 2017, private communication). It should be noted, however, that fluctuations are also predicted from simulations of isolated AGNs (e.g., Novak et al. 2011) and have been observed as well (LaMassa et al. 2015), although this could be related to a tidal disruption event (Merloni et al. 2015). Second, the motion of the gas in the central $\sim \mathrm{kpc}$ of the Western galaxy, as revealed by integralfield spectroscopy, is highly suggestive that the galaxy merger is directly affecting the kinematics of the gas within the nuclear region, providing a direct observational link between the galaxy merger and the change in mass accretion rate onto the black hole.

To better place this AGN pair in the context of galaxy simulations, we estimate the masses of the central SMBHs. For the Eastern galaxy, a broad $\mathrm{H} \alpha$ line was detected, which we use for the $M_{\mathrm{BH}}$ estimation. Greene \& Ho (2005) presented a method for estimating the black hole mass from the width and luminosity of the $\mathrm{H} \alpha$ line. From their Equation (6), given that we measure a width of $4226 \mathrm{~km} \mathrm{~s}^{-1}$ and a luminosity of $2.1 \times 10^{40} \mathrm{erg} \mathrm{s}^{-1}$, we obtain $M_{\mathrm{BH}} \approx 4.6 \times 10^{6} M_{\odot}$. Since no broad line was detected in the Western galaxy, we use the velocity dispersion of the stars in the center of the galaxy to estimate the black hole mass. Using the $M_{\mathrm{BH}}-\sigma_{*}$ relation from Kormendy \& Ho (2013), the calcium triplet measurement implies a black hole mass of $M_{\mathrm{BH}}=3.8_{-2.6}^{+5.1} \times 10^{7} M_{\odot}$ for the Western galaxy. This then implies that the black hole mass ratio of the two galaxies is $10: 1$, which is rather larger than the 4:1 or $2: 1 M_{\mathrm{BH}}$ ratios considered in recent simulations by Gabor et al. (2016).

It is interesting that the AGN in this system with the smallest black hole mass has exhibited the greatest X-ray variability, since it is well known that the variability timescale correlates with black hole mass, i.e., the variability timescale increases with $M_{\mathrm{BH}}$ (e.g., Papadakis 2004). However, these timescales are much shorter $\left(\sim 10^{4} \mathrm{~s}\right)$ than the timescale of the drop in $\mathrm{X}$-ray flux that we have observed.

An observational signature for the drop in $\lambda_{\text {Edd }}$ in the X-ray spectrum of the Eastern source is expected since there is a known correlation between $\lambda_{\text {Edd }}$ and $\Gamma$ (e.g., Shemmer et al. 2006; Risaliti et al. 2009; Brightman et al. 2013, 2016). During the 2004 XMM-Newton observation, the Eastern source had an absorption-corrected $L_{X}$ of $7.5 \times 10^{42} \mathrm{erg} \mathrm{s}^{-1}$. Applying a bolometric correction of 10 (Lusso et al. 2012) implies $L_{\mathrm{Bol}}=7.5 \times 10^{43} \mathrm{erg} \mathrm{s}^{-1}$, which in turn yields $\lambda_{\text {Edd }}=0.12$ for our $M_{\mathrm{BH}}$ estimate. At the time of the Chandra observation, $L_{\mathrm{X}}$ had reduced by a factor of 10 , meaning a decline in $\lambda_{\text {Edd }}$ by the same factor. For the observations in 2014, the X-ray emission from the Eastern nucleus was undetectable, thus $\lambda_{\text {Edd }}$ $\lesssim 0.01$ at that time. From Brightman et al. (2013), $\widetilde{\Gamma}=(0.32 \pm 0.05) \log _{10} \lambda_{\text {Edd }}+(2.27 \pm 0.06)$, thus we would expect $\Delta \Gamma \approx-0.3$ for $\Delta \log _{10} \lambda_{\mathrm{Edd}} \approx-1$. For our analysis presented in Section 3, we tie the $\Gamma$ values to each other for both flux levels. If we perform the same analysis, but with the $\Gamma$ parameter not linked between the observations, we obtain $\Gamma=1.49 \pm 0.12$ for the XMM-Newton observation and $\Gamma=1.53_{-0.57}^{+0.62}$ for the Chandra observation. The uncertainties are therefore too large to constrain $\Delta \Gamma$ at the requisite level.

\section{Summary and Conclusions}

We conducted a multi-wavelength analysis of the galaxy pair ESO 509-IG066 using X-ray, optical, near-IR, and mid-IR data taken between 2004 and 2016. The galaxies in the pair, located at a distance of $150 \mathrm{Mpc}$ with a projected separation of $10.9 \mathrm{kpc}$, were both reported to host an AGN of $L_{\mathrm{X}} \sim 10^{43} \mathrm{erg} \mathrm{s}^{-1}$ by G05 using XMM-Newton data. In an analysis of all available data, we found that since the XMM-Newton observation in 2004, the Eastern nucleus has shown a strong decrease in X-ray flux revealed first by a Chandra observation in 2011. The galaxy remained at this level or lower during a joint $N u S T A R$ and Swift/ XRT observation in 2014. The X-ray emission from the Western source remained relatively constant during this period. Although the $16^{\prime \prime}$ angular separation of the galaxy pair causes significant overlap given the NUSTAR PSF, there is no evidence for the Eastern source in the NUSTAR image from 2014. This argues against a rise in obscuration behind the drop in X-ray flux, unless it is extreme. New Keck/LRIS optical spectroscopy taken after the drop in X-ray flux reveals a broad component to the $\mathrm{H} \alpha$ line from the Eastern nucleus, which also strongly argues against heavy obscuration. We therefore conclude that the AGN has dropped intrinsically in luminosity, most likely due to a decrease in mass accretion rate. From AO-assisted near-infrared integralfield spectroscopy, we find that the kinematics of the gas close to the Western nucleus show evidence that the galaxy merger is having a direct effect close-in to the black hole, providing an observational link between the galaxy merger and the mass accretion rate onto the black hole.

We thank the referee for their constructive input on our manuscript. We also thank Mislav Baloković for introducing us to these interesting galaxies and Jared Gabor for useful discussion. R.J.A. was supported by FONDECYT grant number 1151408. A.M. acknowledges support from the ASI/ INAF grant I/037/12/0-011/13. C.R. acknowledges financial support from the CONICYT-Chile grants "EMBIGGEN" Anillo ACT1101, FONDECYT 1141218, Basal-CATA PFB06/2007 and from the China-CONICYT fund. This work was supported under NASA Contract No. NNG08FD60C, and made use of data from the $N U S T A R$ mission, a project led by the California Institute of Technology, managed by the Jet Propulsion Laboratory, and funded by the National Aeronautics and Space Administration. We thank the NuSTAR Operations, Software, and Calibration teams for support with the execution and analysis of these observations. This research has made use of the NUSTAR Data Analysis Software (NuSTARDAS) jointly developed by the ASI Science Data Center (ASDC, Italy) and the California Institute of Technology (USA). The work presented here was also based on observations obtained with $X M M-N e w t o n$, an ESA science mission with instruments and 
contributions directly funded by ESA Member States and NASA. This publication makes use of data products from the Wide-field Infrared Survey Explorer, which is a joint project of the University of California, Los Angeles, and the Jet Propulsion Laboratory/California Institute of Technology, funded by the National Aeronautics and Space Administration. The data presented herein were obtained at the W.M. Keck Observatory, which is operated as a scientific partnership among the California Institute of Technology, the University of California and the National Aeronautics and Space Administration. The Observatory was made possible by the generous financial support of the W.M. Keck Foundation. The authors wish to recognize and acknowledge the very significant cultural role and reverence that the summit of Maunakea has always had within the indigenous Hawaiian community. We are most fortunate to have the opportunity to conduct observations from this mountain. This research has also made use of data and software provided by the High Energy Astrophysics Science Archive Research Center (HEASARC), which is a service of the Astrophysics Science Division at NASA/GSFC and the High Energy Astrophysics Division of the Smithsonian Astrophysical Observatory. Furthermore, this research has made use of the NASA/IPAC Extragalactic Database (NED), which is operated by the Jet Propulsion Laboratory, California Institute of Technology, under contract with the National Aeronautics and Space Administration. A.M. acknowledges support from the ASI/INAF grant I/037/12/0-011/13, and C.R. acknowledges financial support from the CONICYT-Chile grants "EMBIGGEN" Anillo ACT1101, FONDECYT 1141218, Basal-CATA PFB-06/2007, and from the China-CONICYT fund.

Facilities: NuSTAR, XMM-Newton (pn), Chandra, Swift (XRT), WISE, Keck (LRIS), Keck (OSIRIS).

\section{ORCID iDs}

D. Stern (10 https://orcid.org/0000-0003-2686-9241

M. Koss (i) https://orcid.org/0000-0002-7998-9581

K. Oh (1) https://orcid.org/0000-0002-5037-951X

R. J. Assef (1) https://orcid.org/0000-0002-9508-3667

P. Gandhi (i) https://orcid.org/0000-0003-3105-2615

F. A. Harrison (1) https://orcid.org/0000-0003-2992-8024

H. Jun (i) https://orcid.org/0000-0003-1470-5901

A. Masini (1) https://orcid.org/0000-0002-7100-9366

C. Ricci (1) https://orcid.org/0000-0001-5231-2645

D. J. Walton (1) https://orcid.org/0000-0001-5819-3552

G. Privon (i) https://orcid.org/0000-0003-3474-1125

\section{References}

Alonso, M. S., Lambas, D. G., Tissera, P., \& Coldwell, G. 2007, MNRAS, 375, 1017

Arnaud, K. A. 1996, in ASP Conf. Ser. 101, Astronomical Data Analysis Software and Systems V, ed. G. H. Jacoby \& J. Barnes (San Francisco, CA: ASP), 17

Baumgartner, W. H., Tueller, J., Markwardt, C. B., et al. 2013, ApJS, 207, 19 Berney, S., Koss, M., Trakhtenbrot, B., et al. 2015, MNRAS, 454, 3622 Brightman, M., Masini, A., Ballantyne, D. R., et al. 2016, ApJ, 826, 93 Brightman, M., Silverman, J. D., Mainieri, V., et al. 2013, MNRAS, 433, 2485
Burrows, D. N., Hill, J. E., Nousek, J. A., et al. 2005, SSRv, 120, 165 Cappellari, M., \& Emsellem, E. 2004, PASP, 116, 138

Cardelli, J. A., Clayton, G. C., \& Mathis, J. S. 1989, ApJ, 345, 245 Cash, W. 1979, ApJ, 228, 939

Cusumano, G., La Parola, V., Segreto, A., et al. 2010, A\&A, 524, A64 Di Matteo, T., Springel, V., \& Hernquist, L. 2005, Natur, 433, 604

Drake, A. J., Djorgovski, S. G., Mahabal, A., et al. 2009, ApJ, 696, 870

Ellison, S. L., Patton, D. R., Mendel, J. T., \& Scudder, J. M. 2011, MNRAS, 418, 2043

Gabor, J. M., Capelo, P. R., Volonteri, M., et al. 2016, A\&A, 592, 62 Gandhi, P., Annuar, A., Lansbury, G. B., et al. 2017, MNRAS, 467, 4606

Gehrels, N., Chincarini, G., Giommi, P., et al. 2004, ApJ, 611, 1005

Greene, J. E., \& Ho, L. C. 2005, ApJ, 630, 122

Guainazzi, M., Piconcelli, E., Jiménez-Bailón, E., \& Matt, G. 2005, A\&A, 429, L9

Harrison, F. A., Craig, W. W., Christensen, F. E., Hailey, C. J., \& Zhang, W. W. 2013, ApJ, 770, 103

Hernquist, L. 1989, Natur, 340, 687

Hiroi, K., Ueda, Y., Isobe, N., et al. 2011, PASJ, 63, 677

Hopkins, P. F., Hernquist, L., Cox, T. J., et al. 2005, ApJ, 630, 705

Hopkins, P. F., Hernquist, L., Cox, T. J., et al. 2006, ApJS, 163, 1

Jansen, F., Lumb, D., Altieri, B., et al. 2001, A\&A, 365, L1

Kalberla, P. M. W., Burton, W. B., Hartmann, D., et al. 2005, A\&A, 440, 775

Kauffmann, G., Heckman, T. M., Tremonti, C., et al. 2003, MNRAS, 346,1055

Kewley, L. J., Groves, B., Kauffmann, G., \& Heckman, T. 2006, MNRAS, 372, 961

Kewley, L. J., Heisler, C. A., Dopita, M. A., \& Lumsden, S. 2001, ApJS, 132, 37

Kocevski, D. D., Brightman, M., Nandra, K., et al. 2015, ApJ, 814, 104

Kormendy, J., \& Ho, L. C. 2013, ARA\&A, 51, 511

Koss, M., Mushotzky, R., Treister, E., et al. 2012, ApJL, 746, L22

LaMassa, S. M., Cales, S., Moran, E. C., et al. 2015, ApJ, 800, 144

Larkin, J., Barczys, M., Krabbe, A., et al. 2006, NewAR, 50, 362

Lusso, E., Comastri, A., Simmons, B. D., et al. 2012, MNRAS, 425, 623

Madsen, K. K., Harrison, F. A., Markwardt, C. B., et al. 2015, ApJS, 220, 8

Magdziarz, P., \& Zdziarski, A. A. 1995, MNRAS, 273, 837

Mainzer, A., Bauer, J., Grav, T., et al. 2011, ApJ, 731, 53

Malkan, M. A., Gorjian, V., \& Tam, R. 1998, ApJS, 117, 25

Medling, A. M., U, V., Rich, J. A., et al. 2015, MNRAS, 448, 2301

Merloni, A., Dwelly, T., Salvato, M., et al. 2015, MNRAS, 452, 69

Müller-Sánchez, F., Comerford, J., Stern, D., \& Harrison, F. A. 2016, ApJ, 830,50

Novak, G. S., Ostriker, J. P., \& Ciotti, L. 2011, ApJ, 737, 26

Oke, J. B., Cohen, J. G., Carr, M., et al. 1995, PASP, 107, 375

Osterbrock, D. E. 1981, ApJ, 249, 462

Papadakis, I. E. 2004, MNRAS, 348, 207

Planck Collaboration, Ade, P. A. R., Aghanim, N., et al. 2016, A\&A, 594, A13

Ricci, C., Bauer, F. E., Treister, E., et al. 2017, MNRAS, 468, 1273

Risaliti, G., Salvati, M., Elvis, M., et al. 2009, MNRAS, 393, L1

Rivers, E., Baloković, M., Arévalo, P., et al. 2015a, ApJ, 815, 55

Rivers, E., Risaliti, G., Walton, D. J., et al. 2015b, ApJ, 804, 107

Sanders, D. B., Soifer, B. T., Elias, J. H., et al. 1988, ApJ, 325, 74

Satyapal, S., Ellison, S. L., McAlpine, W., et al. 2014, MNRAS, 441, 1297

Sekiguchi, K., \& Wolstencroft, R. D. 1992, MNRAS, 255, 581

Shemmer, O., Brandt, W. N., Netzer, H., Maiolino, R., \& Kaspi, S. 2006 , ApJL, 646, L29

Silverman, J. D., Kampczyk, P., Jahnke, K., et al. 2011, ApJ, 743, 2

Stern, D., Assef, R. J., Benford, D. J., et al. 2012, ApJ, 753, 30

Strüder, L., Briel, U., Dennerl, K., et al. 2001, A\&A, 365, L18

van Dam, M. A., Bouchez, A. H., Le Mignant, D., et al. 2006, PASP, 118, 310

Van Wassenhove, S., Volonteri, M., Mayer, L., et al. 2012, ApJL, 748, L7

Vazdekis, A., Ricciardelli, E., Cenarro, A. J., et al. 2012, MNRAS, 424, 157

Weisskopf, M. C., Tananbaum, H. D., Van Speybroeck, L. P., \& O’Dell, S. L. 2000, Proc. SPIE, 4012, 2

Wizinowich, P. L., Le Mignant, D., Bouchez, A. H., et al. 2006, PASP, 118,297

Woods, D. F., \& Geller, M. J. 2007, AJ, 134, 527

Wright, E. L., Eisenhardt, P. R. M., Mainzer, A. K., et al. 2010, AJ, 140, 1868 